\title{
Gait spectral index (GSI): a new quantification method for assessing human gait
}

\author{
Rodolphe Héliot ${ }^{1}$, Christine Azevedo-Coste ${ }^{2}$, Laszlo Schwirtlich ${ }^{3}$, Bernard Espiau ${ }^{4}$ \\ ${ }^{1}$ Inria, Montbonnot, France; rodolphe.heliot@gmail.com \\ ${ }^{2}$ Demar Inria/Lirmm, Montpellier, France; $\underline{\text { azevedo@lirmm.fr }}$ \\ ${ }^{3}$ Institute for Rehabilitation “Dr Miroslav Zotovic”, Belgrade, Serbia; laslo@bitsyu.net \\ ${ }^{4}$ Inria, Montbonnot, France; espiau@inrialpes.fr
}

Received 25 November 2009; revised 7 December 2009; accepted 12 December 2009.

\begin{abstract}
This paper introduces a simple, quantitative assessment tool to follow up the recovery of gait. Today, micro-electro-mechanical systems (MEMS) technology provides with small, simple, low-power consuming and easy to don and doff sensors. In our approach we have selected an accelerometer and introduced a new quantity that characterizes the gait pattern in the frequency domain, we term it Gait Spectral Index (GSI). GSI allows assessing gait quality and closely relates to the speed and cadence of gait (dynamics). We have tested the GSI approach to quantify the quality of the gait of healthy young and elderly, and poststroke hemiplegic individuals. We investigated the repeatability and coherence of GSI in healthy individuals (young and elderly) and contrasted this to the post-stroke hemiplegic individuals. We found that high correlation of the GSI with conventional gait parameters. This suggests that GSI, which needs only data from one accelerometer, could be an objective quantitative measure of the quality of the walking thereby a simple yet reliable measure of the recovery of function during neuronrehabilitation.
\end{abstract}

Keywords: Gait Evaluation; Walk Training; Accelerometer; Spectral Analysis

\section{INTRODUCTION}

Clinical methods for gait evaluation consist in standardized functional tests (Barthel Index, Rivermead Mobility Index (Bohannon et al. 1987), Functional Ambulation Category (FAC)) are qualitative; thereby, somewhat subjective because they depend on individual observation skills of the rater. We developed a simple instrument that after appropriate processing provides objective quantita- tive measure of the gait performance. This new instrument is of great importance for clinical use for assessing the gait pattern in all, including elderly and individuals with disabilities (Sekine et al., 2002). The heart of the instrument is the accelerometer that is being mounted on the leg, does not need calibration and provides data that is highly reproducible.

The development of this instrument follows the fast growth of the filed of Micro-electro-mechanical systems (MEMS). The MEMS sensors have been applied for the assessment of physical activities using body-mounted systems (Jasiewicz et al., 2006; Luinge and Veltink, 2004 ; Pappas et al., 2002). MEMS based accelerometers provide a good representation of movement dynamics (Jasiewicz et al., 2006; Hester et al., 2006; Brandes et al., 2005; Luinge and Veltink, 2004). The typical application of multiple accelerometers is for reconstruction of the kinematics of the gait (Wagenaar et al., 1992; Bussmann et al., 2000). Reconstruction of gait kinematics is difficult since the integration of data captured by accelerometers results with drift and substantial errors. The alternative is to use the acceleration data directly as a measure of the quality of gait. Acceleration patterns of trunk have extensively been employed since they have been shown to comprise low intra-variability; thereby suitable for detection of phases of the gait cycle (Ziljstra et al., 2003; Brandes et al., 2006). The reproducibility of trunk accelerations declined as gait speed decreases which is the case when analyzing the gait of elderly and even more of subjects with gait disabilities (Saremi et al., 2006).

Acceleration can be analyzed in the frequency domain. The fundamental frequency is equivalent to the stride frequency, and the harmonics amplitudes vary depending on the gait pattern. It is important to notice that the position of the accelerometer on a body segment does not affect the frequency content of the signal. One of the best confirmations was presented by Waarsing et al. (1996) showing that the power contained in the peaks is inversely proportional to stability performance.

Here, we show that only one accelerometer can be used 
as a good source of data for gait characterization. One of the features of this gait assessment system is that it does not need to be calibrated to each individual, and provides with easy donning and doffing. The major novelty that allows the use of this simple hardware is the Gait Spectral Index (GSI). The GSI is the new measure that characterizes the gait pattern in the frequency domain. We hypothesized that the GSI is highly correlated with the conventional gait parameters. Based on proved hypothesis we performed the experiments in which we compared the GSI determined from analysis of the gait of healthy young and elderly subjects with the GSI determined for the gait of individuals of hemiplegia.

\section{METHODS}

\subsection{Subjects}

Six healthy young (HY) (age 31. 8 years \pm 7.2 ), and ten healthy elderly (HE) (age 67 years \pm 6.9 ) individuals participated in the study to confirm the high correlation of the GSI and conventional gait parameters. 19 post-stroke hemiplegic individuals (SP) (age 58. 6 years \pm 10.18 ) also participated in the study in order to analyze the differences between healthy and pathological gait. Basic tada on study the subjects is summarized in Tables 1-4.

The HY group was formed from volunteers from our laboratory in LIRMM, France. Two subjects, out of ten, in the HE group were recruited in an elderly cultural association while the other eight from a local waling club.

Hemiplegic individuals were recruited from the inpatient population of the Institute for Rehabilitation "Dr Miroslav Zotovic" in Belgrade. The hemiplegic gait abilities were assessed by using the Functional Ambulation Category scale (Holden et al, 1984). The FAC scale has five grades: 1 - person needs to be physically supported for any ambulation (the worst), and 5 - the person can walk independently anywhere (the best). All hemiplegic individuals could walk with their usual walking aid (13 patients used cane or tripod, 4 patients had also an ankle or foot orthosis) as shown in Tables 3 and 4.

The (SP) group was divided into two sub-groups: (SP1) includes the eight individuals with a 5 FAC rank and (SP2) with the 11 remaining individuals.

This study was approved by the local ethics Committee, and all study participants signed the informed consent.

\subsection{Equipment}

We used a uniaxial-accelerometer (ADXL-203, Analog Devices) positioned on the shank close to the ankle. The accelerometer axis was directed along the shank with the positive direction pointing upwards (Figure 1). The alignment was performed visually, except from this constraint, no specific care was needed for the positioning of the accelerometer. In the case of hemiplegic individuals the accelerometer was positioned on the non-paretic leg. In order to determine the correlation with the conventional gait parameters subjects were also equipped with two 3-contact point insoles used to detect gait phases, gait cadence and assess the averaged stride length when associated to chronometric recordings. The sampling rate was 100 samples per second based on known low frequency content of the signal.

\subsection{Protocol}

Subjects were asked to walk 10 meters at their normal self-paced speed. Depending on the individual ability to

Table 1. Healthy young subject group (HY) gait description.

\begin{tabular}{ccc}
\hline$\#$ & Age & Cadence \\
\hline 6 & 30 & 0.90 \\
1 & 46 & 0.96 \\
2 & 28 & 0.78 \\
3 & 32 & 1.05 \\
5 & 26 & 0.99 \\
4 & 29 & 0.84 \\
\hline Mean & $\mathbf{3 1 . 8}$ & $\mathbf{0 . 9 2}$ \\
Std & $\mathbf{7 . 2}$ & $\mathbf{0 . 1}$ \\
\hline
\end{tabular}

Table 2. Healthy elderly subject group (HE) gait description.

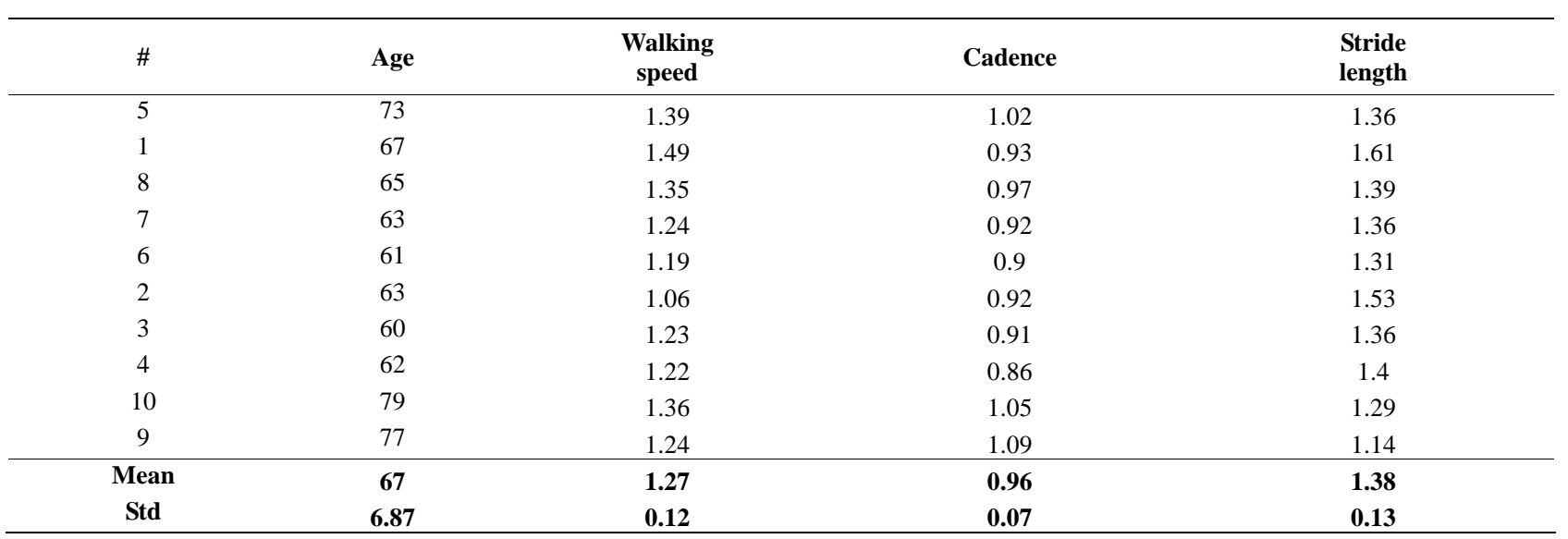


Table 3. Stroke patient group 1 (SP1) gait description.

\begin{tabular}{cccccccc}
\hline$\#$ & Age & FAC & Walking aid & $\begin{array}{c}\text { Walking } \\
\text { speed }\end{array}$ & Cadence & $\begin{array}{c}\text { Stride } \\
\text { length }\end{array}$ & SI \\
\hline 4 & 54 & 4 & Cane & 0.3 & 0.44 & 0.68 & 15.47 \\
3 & 69 & 4 & Cane & 0.38 & 0.56 & 0.68 & 27.61 \\
6 & 71 & 3 & Tripod & 0.13 & 0.29 & 0.46 & 27.47 \\
13 & 53 & 4 & AFO+tripod+physio & 0.1 & 0.22 & 0.48 & 16.99 \\
5 & 67 & 1 & AFO+tripod & 0.12 & 0.32 & 0.4 & 9.83 \\
16 & 77 & 3 & Tripod & 0.17 & 0.33 & 0.51 & 17.06 \\
17 & 59 & 2 & Tripod+physio & 0.11 & 0.2 & 0.58 & 22.18 \\
8 & 47 & 3 & Tripod & 0.29 & 0.39 & 0.75 & 66.57 \\
11 & 46 & 3 & Cane & 0.07 & 0.33 & 0.24 & 3.71 \\
7 & 77 & 4 & AFO+tripod & 0.2 & 0.54 & 0.72 & 6.54 \\
9 & 58 & 4 & Tripod & 0.17 & 0.28 & 0.6 & 11.65 \\
\hline Mean & $\mathbf{6 1 . 6 4}$ & $\mathbf{3 . 1 8}$ & & $\mathbf{0 . 1 9}$ & $\mathbf{0 . 3 5}$ & $\mathbf{0 . 5 5}$ & $\mathbf{2 0 . 4 6}$ \\
Std & $\mathbf{1 1 . 2 0}$ & $\mathbf{0 . 9 8}$ & & $\mathbf{0 . 1 0}$ & $\mathbf{0 . 1 2}$ & $\mathbf{0 . 1 5}$ & $\mathbf{1 7 . 1 5}$ \\
\hline
\end{tabular}

Table 4. Stroke patient group 2 (SP2) gait description.

\begin{tabular}{cc}
\hline$\#$ & GSI \\
\hline 6 & 1.57 \\
1 & 1.46 \\
2 & 1.42 \\
3 & 1.40 \\
5 & 1.28 \\
4 & 0.92 \\
\hline Mean & $\mathbf{1 . 3 4}$ \\
Std & $\mathbf{0 . 2 3}$ \\
\hline
\end{tabular}

walk for extended period of time, the data between four and eight trials were collected for each subject.

\subsection{Symmetry Index}

The symmetry index (SI) can be calculated for swing and stance phases using the following formula (Robinson et al., 1987):

$$
\mathrm{SI}[\%]=200\left(\mathrm{~T}_{\text {nonparetic }}-\mathrm{T}_{\text {paretic }}\right) /\left(\mathrm{T}_{\text {paretic }}+\mathrm{T}_{\text {nonparetic }}\right)
$$

$\mathrm{T}_{\text {paretic }}$ and $\mathrm{T}_{\text {nonparetic }}$ are the durations of stance or swing phases for paretic and non paretic legs. SI can be positive or negative and the perfect symmetry index is $\mathrm{SI}=0$.

\subsection{Spectral Analysis and Gait Spectral Index}

The recorded acceleration was transformed using the Fast Fourier transform (FFT) to obtain the frequency spectrum of the signal. There was no averaging; the spectrums were computed over the whole trial duration (10 meters). The gait spectral index (GSI) was defined as a ratio between the power of the second harmonic component with respect the power of the fundamental component.

$$
\begin{gathered}
\text { GSI }=\text { Power_of_second_harmonic/ } \\
\text { Power_of_fundamental }
\end{gathered}
$$

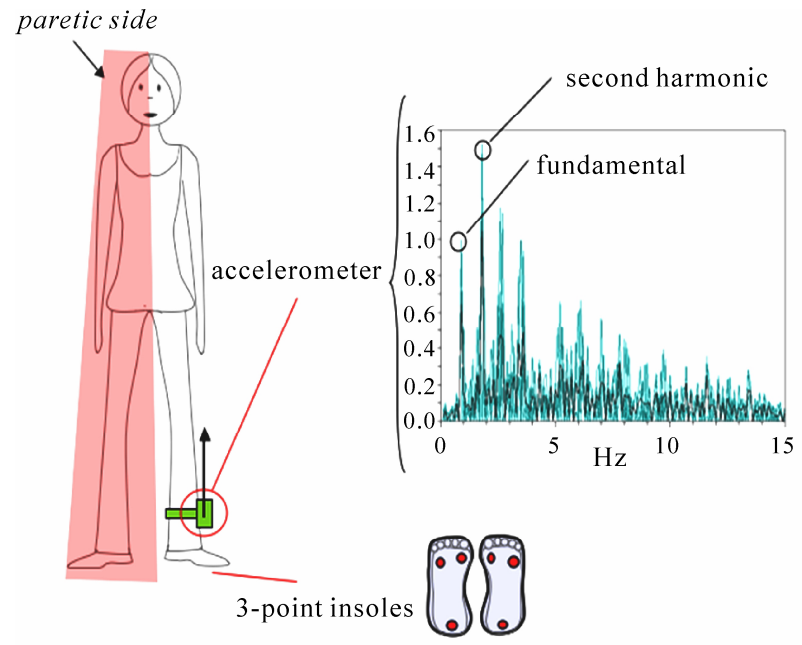

Figure 1. Protocol description.

\section{Results}

In Tables 1-4 we reported the gait characteristics (walking speed, cadence, stride length, symmetry index) measured for each of the four groups.

Figure 2 shows the spectrums corresponding to one individual of each population. Spectrums from a given subject are highly reproducible from a trial to another and spectrums for each of the groups are significant in terms of harmonic repartitions (Tables 7 and 8). The computed GSI for the three groups are reported in tables 5 to 8 . The aim of healthy group individuals was to compute GSI, therefore we did not analyze all the gait characteristics for these two populations.

\subsection{Patients}

In patient group GSI index varies from 0.52 to 1.69 (tables 7 and 8) with a low intra variation (average standard deviation 0.16 ). The spectrum reproducibility is good as 
stated by the low intra variability associated to fundamental and $2^{\text {nd }}$ harmonic amplitudes (tables 7 and 8). For each individual, we have also estimated the correlation between each trial spectrum and the average spectrum for this given individual. The good correlation ( $\mathrm{r}=0.78$ ) validates the hypothesis of the spectrum reproducibility. There is a moderate correlation between GSI and FAC rank ( $\mathrm{r}=$ 0.66). In figure 3 we plotted the GSI values obtained for the two patient groups in function of FAC rank. In table 7, the patients are classified in terms of GSI index values. The higher GSI values correspond to best walkers in FAC quotation (level 5-group SP1), they vary from 1.22 to 1.69. The mean value is 1.21 . In SP2 group, GSI varies from 0.52 to 0.89 (table 8 ). For lower levels, the GSI does not seem to allow to discriminate between the different levels. The mean value is 0.71 .

There is a good correlation between GSI and gait speed $(\mathrm{r}=0.81)$ and gait cadence $(\mathrm{r}=0.74)$. Correlation between GSI and stride length is moderate $(\mathrm{r}=0.66)$. Correlation with symmetry index is not significant. The mean value of symmetry index is $21 \%$.

It is interesting to notice that FAC rank and symmetry index are not correlated $(\mathrm{r}=0.03)$. FAC rank is correlated with gait speed $(r=0.7)$, gait cadence $(r=0.72)$ and stride length $(\mathrm{r}=0.66)$.

\subsection{Elderly}

In elderly subject group GSI index varies from 0.4 to 1.3 (table 5). The mean value is 1.02. In healthy elderly group, only stride length appeared to be significant in terms of correlation with GSI $(\mathrm{r}=0.72)$. The two older subjects $(77$ and 79) have the lower GSI scores.

\subsection{Young}

In young subject group GSI index varies from 0.92 to 1.57 (table 6). The mean value is 1.34 .

\section{Discussion}

The results presented document that the spectrum of the shank acceleration is highly reproducible from trial to trial in the same subject and can be considered as an individual signature pattern. We noticed the presence of strong high frequencies components in the spectrums of healthy and patient best walkers. High frequencies components are likely present due to the richer dynamics of healthy gait compared to the gait of hemiplegic and poor walkers.

The proposed GSI ratio compares fundamental amplitude with second harmonic amplitude; it correlates with gait speed which is classically considered as an indirect measurement of gait quality. It is important to notice that to be valuable speed should be evaluated through distances long enough to assess adaptation and gait efficiency.

The low correlation between FAC score and GSI should be counterbalanced by the fact that the higher GSI values correspond to best classification in FAC. But. FAC classes are not varying linearly with gait quality and the number of patients in each of the categories is not similar enough to conclude.

The GSI scores in elderly population are higher compared to our expectations. We suggest that this is due to the fact that the population included in the study was mainly recruited in a gait club. Hence, they are not representative for typical aged walkers with limited sensory-motor capacity.

Nor GSI, nor FAC are correlated with symmetry of gait in patients. This should be an improvement of GSI in future.

\section{CONCLUSIONS}

The spectral analysis of the shank acceleration, in the direction aligned with the shank can characterize the gait. Two main observations can be made: 1) dealing with a given subject: high reproducibility of spectrums from trial to trial, and 2) presence of strong high frequencies components in the spectrums of healthy and patients who are good walkers.

We found out that placing one accelerometer on the "healthy" leg of post-stroke hemiplegic patient allows assessing the effects of gait reeducation. The instrumentation and processing that we described are simple and they comprise light and non expensive setup, including wireless communication of sensory output to computer.

At this point the GSI index is mainly correlated with gait speed and gait cadence and better than what FAC rank. This is an interesting result as our approach is technically less constraining than classical methods to

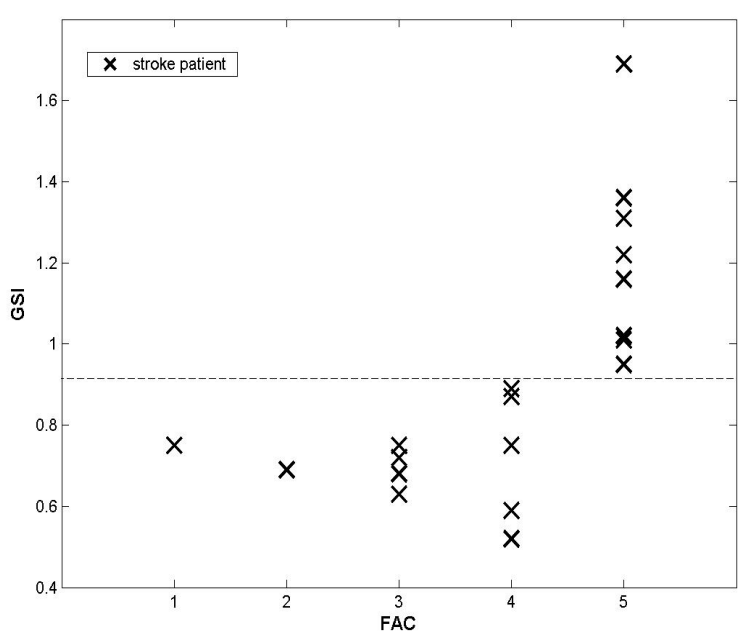

Figure 1. Hemiparetic stroke GSI. 


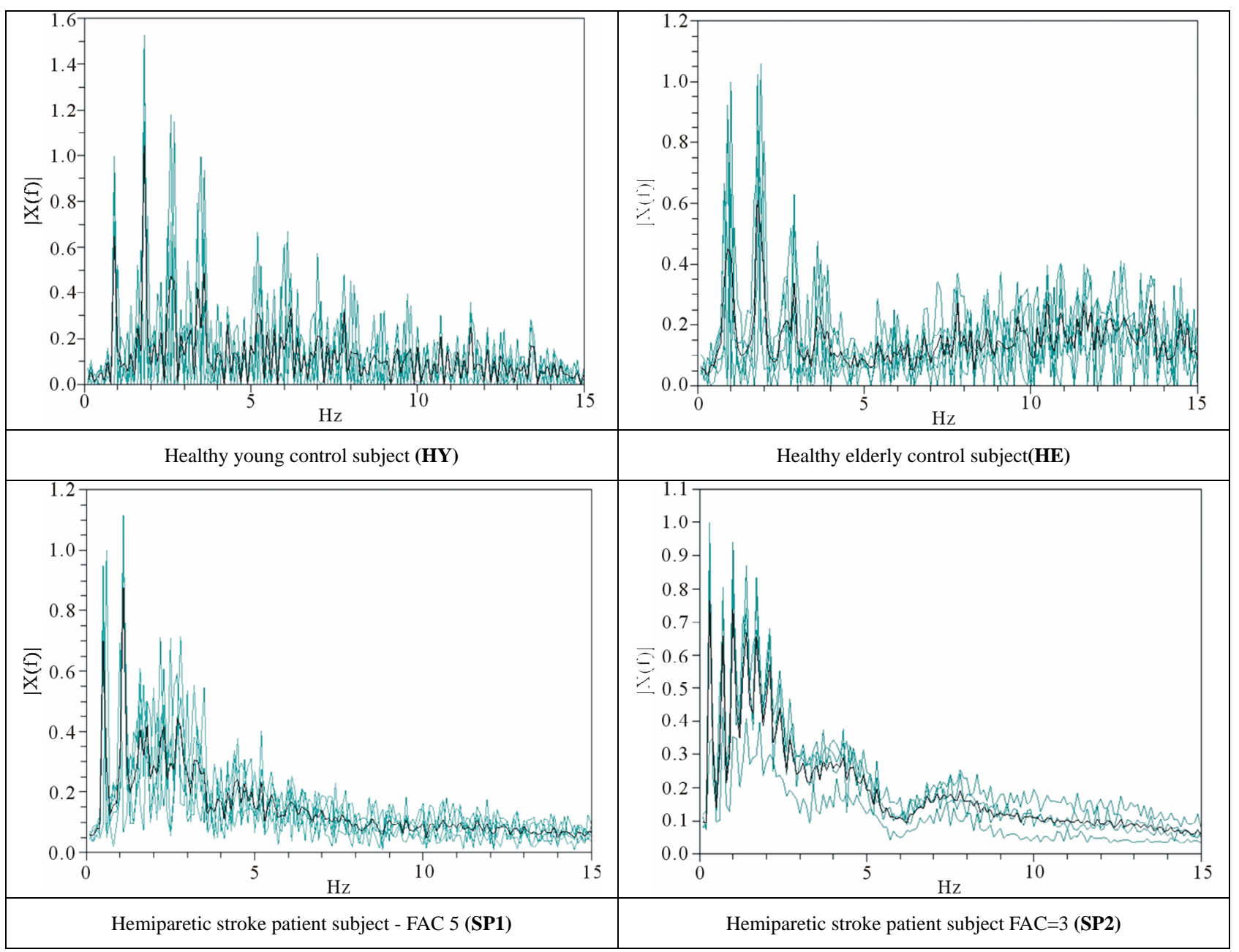

Figure 2. Accelerometer signals spectrums examples for all 4 groups. The values on the vertical axis are normalized relatively to the amplitude of the fundamental amplitude. The value of the 2 nd harmonic therefore corresponds to GSI.

Table 5. Healthy young subject group (HY) mean value of the GSI index over different trials for each subject for comparison purposes.

\begin{tabular}{cc}
\hline$\#$ & GSI \\
\hline 6 & 1.57 \\
1 & 1.46 \\
2 & 1.42 \\
3 & 1.40 \\
5 & 1.28 \\
4 & 0.92 \\
\hline Mean & $\mathbf{1 . 3 4}$ \\
Std & $\mathbf{0 . 2 3}$ \\
\hline
\end{tabular}

assess the gait speed and cadence (chronometer, insoles).

The GSI could be applied not only for the individuals who participated in this study, but in many other types of pathological gait. The GSI needs then to be improved and adapted to meet the specific pathologies and give object-
Table 6. Healthy elderly subject group (HE) mean value of the GSI index over different trials for each subject for comparison purposes.

\begin{tabular}{cc}
\hline$\#$ & GSI \\
\hline 5 & 1.3 \\
1 & 1.29 \\
8 & 1.2 \\
7 & 1.19 \\
6 & 1.12 \\
2 & 1.11 \\
3 & 0.98 \\
4 & 0.88 \\
10 & 0.78 \\
9 & 0.4 \\
\hline Mean & $\mathbf{1 . 0 2 5}$ \\
Std & $\mathbf{0 . 2 8}$ \\
\hline
\end{tabular}

tive measures of the improvement. 
Table 7. Stroke patient group 1 (SP1) GSI indexes and spectral analysis results.

\begin{tabular}{cccccccc}
\hline & \multicolumn{2}{c}{$\begin{array}{c}\text { GSI } \\
\text { GSI }\end{array}$} & \multicolumn{2}{c}{$\begin{array}{c}\text { Fundamental } \\
\text { amplitude }\end{array}$} & \multicolumn{2}{c}{$\begin{array}{c}\mathbf{2}^{\text {nd }} \text { harmonic } \\
\text { amplitude }\end{array}$} & $\begin{array}{c}\text { Spectrum } \\
\text { correlation }\end{array}$ \\
\cline { 2 - 8 } & mean & std & mean & std & mean & std & \\
\hline 2 & 1.69 & 0.10 & 0.85 & 0.07 & 1.46 & 0.20 & 0.79 \\
1 & 1.36 & 0.20 & 0.70 & 0.25 & 0.93 & .027 & 0.81 \\
20 & 1.31 & 0.22 & 0.82 & 0.16 & 1.09 & 0.27 & 0.79 \\
15 & 1.22 & 0.21 & 0.94 & 0.05 & 1.15 & 0.19 & 0.51 \\
14 & 1.16 & 0.09 & 0.89 & 0.13 & 1.03 & 0.21 & 0.77 \\
19 & 1.02 & 0.09 & 0.93 & 0.08 & 0.96 & 0.14 & 0.67 \\
10 & 1.01 & 0.09 & 0.89 & 0.08 & 0.90 & 0.11 & 0.79 \\
18 & 0.95 & 0.14 & 0.93 & 0.03 & 0.89 & 0.16 & 0.79 \\
\hline Mean & $\mathbf{1 . 2 2}$ & $\mathbf{0 . 1 4}$ & $\mathbf{0 . 8 7}$ & $\mathbf{0 . 1 1}$ & $\mathbf{1 . 0 5}$ & $\mathbf{0 . 1 9}$ & $\mathbf{0 . 7 9}$ \\
Std & $\mathbf{0 . 2 4}$ & $\mathbf{0 . 0 5}$ & $\mathbf{0 . 0 8}$ & $\mathbf{0 . 0 7}$ & $\mathbf{0 . 1 8}$ & $\mathbf{0 . 0 5}$ & $\mathbf{0 . 1 0}$ \\
\hline
\end{tabular}

Table 8. Stroke patient group 2 (SP2) GSI indexes and spectral analysis results.

\begin{tabular}{|c|c|c|c|c|c|c|c|}
\hline \multirow[t]{2}{*}{$\#$} & \multicolumn{2}{|c|}{$\begin{array}{l}\text { GSI } \\
\text { GSI }\end{array}$} & \multicolumn{2}{|c|}{$\begin{array}{l}\text { Fundamental } \\
\text { amplitude }\end{array}$} & \multicolumn{2}{|c|}{$\begin{array}{l}2^{\text {nd }} \text { harmonic } \\
\text { amplitude }\end{array}$} & \multirow[t]{2}{*}{$\begin{array}{c}\text { Spectrum } \\
\text { correlation }\end{array}$} \\
\hline & mean & std & mean & & mean & std & \\
\hline 4 & 0.89 & 0.15 & 0.90 & 0.06 & 0.80 & 0.16 & 0.80 \\
\hline 3 & 0.87 & 0.10 & 0.92 & 0.05 & 0.81 & 0.13 & 0.85 \\
\hline 6 & 0.75 & 0.25 & 0.71 & 0.31 & 0.47 & 0.14 & 0.92 \\
\hline 13 & 0.75 & 0.45 & 0.61 & 0.42 & 0.35 & 0.15 & 0.63 \\
\hline 5 & 0.75 & 0.12 & 0.85 & 0.07 & 0.64 & 0.14 & 0.89 \\
\hline 16 & 0.72 & 0.13 & 0.91 & 0.07 & 0.66 & 0.10 & 0.94 \\
\hline 17 & 0.69 & 0.24 & 0.53 & 0.40 & 0.32 & 0.14 & 0.49 \\
\hline 8 & 0.68 & 0.19 & 0.75 & 0.19 & 0.51 & 0.16 & 0.80 \\
\hline 11 & 0.63 & 0.12 & 0.64 & 0.37 & 0.37 & 0.18 & 0.65 \\
\hline 7 & 0.59 & 0.14 & 0.75 & 0.25 & 0.43 & 0.14 & 0.95 \\
\hline 9 & 0.52 & 0.04 & 0.88 & 0.11 & 0.46 & 0.07 & 0.96 \\
\hline Mean & 0.71 & 0.17 & 0.77 & 0.21 & 0.53 & 0.14 & 0.81 \\
\hline Std & 0.11 & 0.10 & 0.13 & 0.14 & 0.17 & 0.03 & 0.15 \\
\hline
\end{tabular}

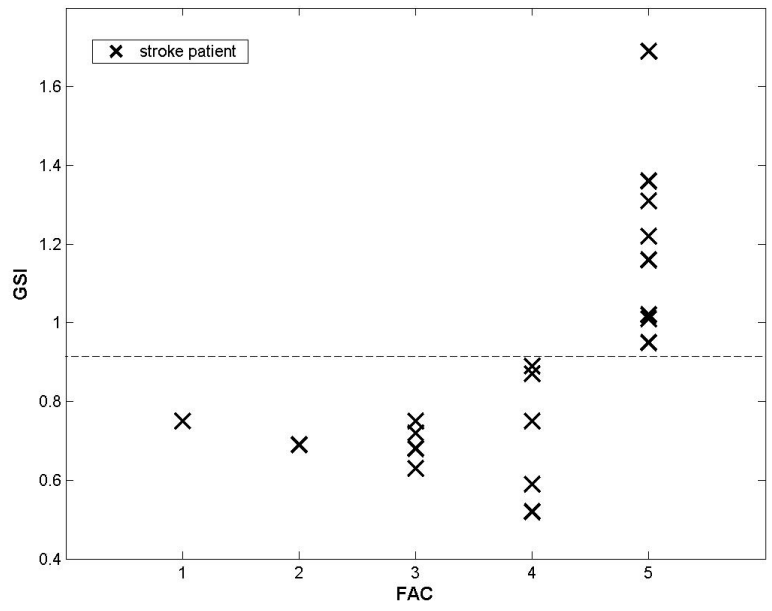

Figure 3. Hemiparetic stroke GSI.

In the future, we propose to improve GSI in order to include gait symmetry parameter. It would also be possible to give different weights to different gait quality criteria within GSI.

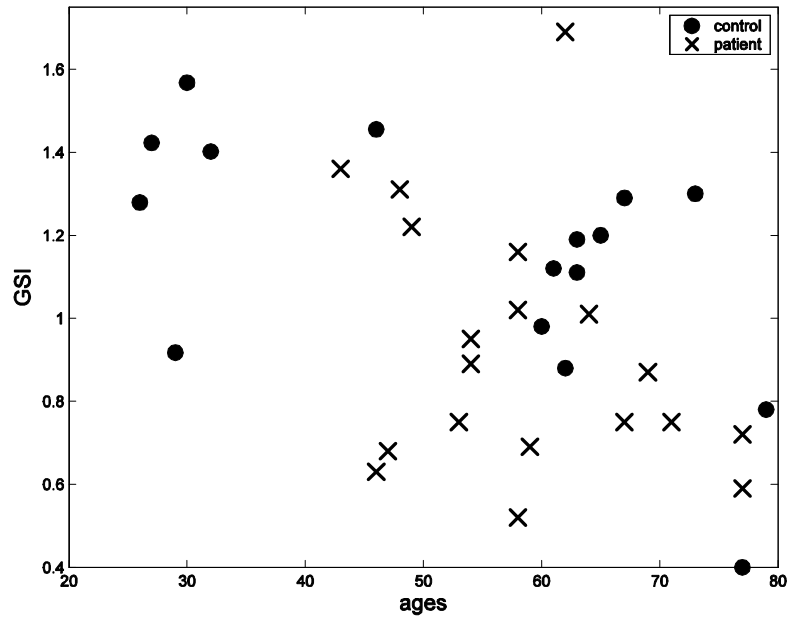

Figure 4. GSI / subject ages.

The low intra variability of this spectral analysis could easily be employed to assess the gait improvement of each individual patient. A low GSI score could alert the therapist on possible pathological problems. 


\section{REFERENCES}

[1] Bohannon, R.W., Smith, M.B. (1987) Interrater reliability of modified Ashworth scale of muscle spasticity. Phys Ther; 67, 206-7.

[2] Bonnet, S. and Heliot, R. (2007) A magnetometer-based approach for studying human movements to appear. IEEE Trans. Biomedical Engineering.

[3] Brandes, M., Zijlstra, W., Heikens, S.R., Lummel, R. Van and Rosenbaum, D. (2005) Accelerometry based assessment of gait parameters in children. Gait \& Posture, 24, 482-486.

[4] Bussmann, J.B. and Damen, L. and Stam, H.J. (2000) Analysis and decomposition of signals obtained by thigh-fixed uni-axial accelerometry during normal gait. Med Biol Eng Comput, 38, 632-638.

[5] Hester, T., Hughes, R., Sherrill, D., Knorr, B., Akay, M., Stein J., and Bonato, P. (2006) Using wearable sensors to measure motor abilities following stroke. International Workshop on Wearable and Implantable Body Sensor Networks, Massachusetts.

[6] Holden, M.K., Gill, K.M., Magliozzi, M.R. and et al. (1984) Clinical gait assessment in the neurologically impaired, reliability and meaningfulness. Physical Therapy, 64, 35-40.

[7] Jasiewicz, J.M., Allum, J. H.J., Middleton, J. W., Barriskill, A., Condie, P., Purcell, B. and Che Tin Li, R.C.T. (2006) Gait event detection using linear accelerometers or angular velocity transducers in able-bodied and spinal-cord injured individuals. Gait \& Posture, 24, 502-509.

[8] Lee, J.A. and Cho, S.H. and Lee, J.W. and Lee, K.H. and. Yang, H.K. (2007) Wearable Accelerometer System for Measuring the Temporal Parameters of Gait. Engineering in Medicine and Biology Society EMBS, 483-486.

[9] Luinge, H. and Veltink, P. (2004) Inclination measurement of human movement using a $3 \mathrm{~d}$ accelerometer with autocalibration. IEEE Trans. Neural Sys. and Rehabil. Eng., 12, 112-121.

[10] Montoye, H.J., Washburn, R., Servais, S., Ertl, A., Webster, J.G. and Nagle, F.J. (1983) Estimation of energy expenditure by a portable accelerometer. Medi. Sci. Sport Exercise, 15, 403-407.

[11] Pappas, I.P.I., Keller, T. and Mangold, S. A reliable, gyroscope based gait phase detection sensor embedded in a shoe insole. (2002) In Proceedings of IEEE Sensors 002, First IEEE International Conference on Sensors, 2, 1085-1088.

[12] Robinson, R.O., Herzog, W., Bigg, B.M. (1987) Use of platform variables to quantify the effects of chiropractors manipulation on gait symmetry. $J$ Manipulative Physiol Ther. 10, 172-176.

[13] Roth, E.J. Merbitz, C., Mroczek, K., Dugan, and Suh, W.W. (1997) Hemiplegic gait. relationships between gait speed and other temporal parameters. Am J Phys Med Rehabil., 76(2) , 128-133.

[14] Saremi, K., Marehbian, J., Yan, X., Regnaux, J.P., Elashoff, R., Bussel, B. and Dobkin, B.H. (2006) Reliability and validity of bilateral thigh and foot accelerometric measures in healthy and hemiparetic subjects. The American Society of Neurorehabilitation, 20(2).

[15] Sekine, M., Tamura, Akay, M.T., Fujimoto, T., Togawa, and Fukui, Y. (2002) Discrimination of gait patterns using wavelet-based fractal analysis. IEEE transactions on Neural Systems and Rehabilitation Engineering, 10-3, 188-196.

[16] Waarsing, J.H., Mayagoitia, R.E. and Veltink, P.H (1996) Quantifying the stability of gait using accelerometers. In 18th Annual International Conference of the IEEE Engineering in Medicine and Biology Society, Amsterdam. utilisation d'accéléros pour quantifier la marche.

[17] Wagenaar, R.C. and Beek, W.J. (1992) Hemiplegic gait, a kinematic analysis using gait speed as a basis. J Biomech.25, 1007-1115.

[18] Ziljstra, W. and Hof, A.L. (2003) Assessment of spatio-temporal gait parameters from trunk accelerations during human gait. Gait \& Posture, 4, 212-221. 